\title{
PROBABILITY \\ AND \\ SCHRÖDINGER'S \\ MECHANICS
}


This page is intentionally left blank 


\section{PROBABILITY}

\section{AND \\ SCHRÖDINGER'S \\ MECHANICS}

\section{David B. Cook}

Department of Chemistry, University of Sheffield 


\section{Published by}

World Scientific Publishing Co. Pte. Ltd.

5 Toh Tuck Link, Singapore 596224

USA office: Suite 202, 1060 Main Street, River Edge, NJ 07661

UK office: 57 Shelton Street, Covent Garden, London WC2H 9HE

\section{British Library Cataloguing-in-Publication Data}

A catalogue record for this book is available from the British Library.

\section{PROBABILITY AND SCHRÖDINGER'S MECHANICS}

Copyright $(2002$ by World Scientific Publishing Co. Pte. Ltd.

All rights reserved. This book, or parts thereof, may not be reproduced in any form or by any means, electronic or mechanical, including photocopying, recording or any information storage and retrieval system now known or to be invented, without written permission from the Publisher.

\footnotetext{
For photocopying of material in this volume, please pay a copying fee through the Copyright Clearance Center, Inc., 222 Rosewood Drive, Danvers, MA 01923, USA. In this case permission to photocopy is not required from the publisher.
}

ISBN 981-238-191-0

This book is printed on acid-free paper.

Printed in Singapore by Mainland Press 


\begin{abstract}
"But the chemists and the whole class of mechanics and empirics, should they have the temerity to attempt contemplation and philosophy, being accustomed to meticulous subtlety in a few things, they twist by extraordinary means all the rest into conformity with them and promote opinions more odious and unnatural than those advanced by the very rationalists."
\end{abstract}

- Francis Bacon, Preface to Natural History, 1609 (my emphasis) 
This page is intentionally left blank 


\section{Contents}

Preface xiii

Organisation xvii

$\begin{array}{lll}\text { Part } 1 \text { Preliminaries } & 1\end{array}$

Chapter 1 Orientation and Outlook 3

1.1. General Orientation . . . . . . . . . . . . . . . . 3

1.2. Materialism . . . . . . . . . . . . . . . 4

1.3. Materialism and Realism . . . . . . . . . . . 8

1.4. $\operatorname{Logic} \ldots \ldots \ldots \ldots \ldots$

1.5. Mathematics . . . . . . . . . . . . . . . 12

1.6. Reversing Abstraction . . . . . . . . . . . . 13

1.7. Definitions, Laws of Nature and Causality . . . . . . . 15

1.8. Foundations . . . . . . . . . . . . . . 18

1.9. Axioms . . . . . . . . . . . . . . . . 20

1.10. An Interpreted Theory . . . . . . . . . . . . . . 21

$\begin{array}{lll}\text { Part } 2 & \text { Probabilities } & 23\end{array}$

Chapter 2 Simple Probabilities 25

2.1. Colloquial and Mathematical Terminology . . . . . . . . . 26

2.2. Probabilities for Finite Systems . . . . . . . . . . . 27

2.2.1. An Example: The Faces of a Cube . . . . . . . . 29

2.2.2. Dice: Statistical Methods of Measure . . . . . . . 31 
2.2.3. Loaded Dice: Statistical Methods of Measure . . . . . 34

2.2.4. Standard Dice and Conservation Laws . . . . . . . 35

2.3. Probability and Statistics . . . . . . . . . . . . . 39

2.3.1. An Extreme Example . . . . . . . . . . . . . . 40

2.4. Probabilities in Deterministic Systems . . . . . . . . . 41

2.5. The Referent of Probabilities and Measurement . . . . . . . 45

2.5.1. Single System or Ensemble? . . . . . . . . . . . 47

2.5.2. The Collapse of the Distribution . . . . . . . . . 49

2.5.3. Hidden Variables . . . . . . . . . . . . . 50

2.6. Preliminary Summary . . . . . . . . . . . . . 51

Chapter 3 A More Careful Look at Probabilities 53

3.1. Abstract Objects . . . . . . . . . . . . . . . 53

3.2. States and Probability Distributions . . . . . . . . 55

3.2.1. The Propensity Interpretation . . . . . . . . . . 56

3.3. The Formal Definition of Probability . . . . . . . . . 58

3.3.1. A Premonition . . . . . . . . . . . . . . . . 62

3.4. Time-Dependent Probabilities . . . . . . . . . . . . 63

3.5. Random Tests . . . . . . . . . . . . . . . . . . . 66

3.6. Particle-Distribution Probabilities . . . . . . . . . 67

$\begin{array}{lll}\text { Part } 3 \text { Classical Mechanics } & 69\end{array}$

Chapter 4 The Hamilton-Jacobi Equation 71

4.1. Historical Connections . . . . . . . . . . . . . . 71

4.2. The H-J Equation . . . . . . . . . . . . . . . . . 73

4.3. Solutions of the H-J Equation . . . . . . . . . . . . 76

4.3.1. Cartesian Coordinates . . . . . . . . . . . . 78

4.3.2. Spherical Polar Coordinates . . . . . . . . . . 79

4.3.3. Comparisons . . . . . . . . . . . . . . . . 81

4.3.4. Cylindrical Coordinates . . . . . . . . . . . 83

4.4. Distribution of Trajectories . . . . . . . . . . 84

4.5. Summary . . . . . . . . . . . . . . . . 86

$\begin{array}{llr}\text { Appendix 4.A } & \text { Transformation Theory } & 89\end{array}$

Chapter 5 Angular Momentum 99

5.1. Coordinates and Momenta . . . . . . . . . . . . . . . . 99

5.2. The Angular Momentum "Vector" . . . . . . . . . . . . . . 101 
5.3. The Poisson Brackets and Angular Momentum . . . . . . 106

5.4. Components of the Angular Momentum "Vector" . . . . . . 107

5.5. Conclusions for Angular Momentum . . . . . . . . . . . 109

$\begin{array}{lll}\text { Part } 4 \text { Schrödinger's Mechanics } & 111\end{array}$

Chapter 6 Prelude: Particle Diffraction 113

6.1 . History . . . . . . . . . . . . . . . . . . . . . 113

6.1.1. The Experiment . . . . . . . . . . . . . . 114

6.1.2. The Explanations . . . . . . . . . . . . . . . . 114

6.2. The Wave Theory . . . . . . . . . . . . . 115

6.3. The Particle Theory . . . . . . . . . . . . . 116

6.4. A Simple Case . . . . . . . . . . . . . . . . . . . . 118

6.5. Experimental Verification . . . . . . . . . . . 120

6.6. The Answer to a Rhetorical Question . . . . . . . . . 120

6.7. Conclusion . . . . . . . . . . . . . . . . . . . . 121

Chapter 7 The Genesis of Schrödinger's Mechanics 123

7.1. Lagrangians, Hamiltonians, Variation Principles . . . . . . . 123

7.1.1. Equations and Identities . . . . . . . . . . . 125

7.2. Replacing the Hamilton-Jacobi Equation . . . . . . . . . 126

7.3. Generalising the Action $S \ldots \ldots \ldots \ldots \ldots \ldots$

7.3.1. Changing the Notation for Action . . . . . . . . . 129

7.3.2. Interpreting the Change . . . . . . . . . 131

7.4. Schrödinger's Dynamical Law . . . . . . . . . . . . . 134

7.4.1. Position Probability and Energy Distributions . . . . 135

7.4.2. The Schrödinger Condition . . . . . . . . . . 136

7.5. Probability Distributions? . . . . . . . . . . . . 139

7.6. Summary of Basic Principles . . . . . . . . . . . . . . 142

Chapter 8 The Schrödinger Equation 147

8.1. The Variational Derivation . . . . . . . . . . . . . . 147

8.2. Some Interpretation . . . . . . . . . . . . . . . . . 152

8.3. The Boundary Conditions . . . . . . . . . . . 156

8.4. The Time-Independent Schrödinger Equation . . . . . . . . 158

Appendix 8.A Schrödinger's First Paper of $1926 \quad 161$ 
Chapter 9 Identities: Momenta and Dynamical Variables 179

9.1. Momentum Definitions and Distributions . . . . . . . . . . . 179

9.2. Abstract Particles of Constant Momentum . . . . . . . . . . 180

9.3. Action and Momenta in Schrödinger's Mechanics . . . . . . 182

9.4. Momenta and Kinetic Energy . . . . . . . . . . . . . 186

9.5. Boundary Conditions . . . . . . . . . . . . . . . . . . . . 189

9.5.1. Constant Momenta and Kinetic Energy . . . . . . . . 190

9.5.2. Solution of the Schrödinger Equation . . . . . . . . 191

9.6. The "Particle in a Box" and Cyclic Boundary Conditions . . . 192

Chapter 10 Abstracting the Structure 195

10.1. The Idea of Mathematical Structure . . . . . . . . . . . 195

10.1.1. A Pitfall of Abstraction: The Momentum Operator . 198

10.2. States and Hilbert Space . . . . . . . . . . . . . . . . . 201

10.3. The Real Use of Abstract Structures . . . . . . . . . . . 204

$\begin{array}{lll}\text { Part } 5 & \text { Interpretation from Applications } & 207\end{array}$

Chapter 11 The Quantum Kepler Problem 209

11.1. Two Interacting Particles . . . . . . . . . . . . . . . . . . 210

11.2. Quantum Kepler Problem in a Plane . . . . . . . . . . . 211

11.3. Abstract and Concrete Hydrogen Atoms . . . . . . . . . . . . 212

11.4. The Kepler Problem in Three Dimensions . . . . . . . . . . . 214

11.5. The Separation of the Schrödinger Equation . . . . . . . . 216

11.6. Commuting Operators and Conservation . . . . . . . . . 218

11.7. The Less Familiar Separations . . . . . . . . . . . . . . 221

11.7.1. The Everyday Solutions . . . . . . . . . . . . . 223

11.8. Conservation in Concrete and Abstract Systems . . . . . . . 223

11.9. Conclusions from the Kepler Problem . . . . . . . . . . . . 227

11.9.1. Concrete Objects and Symmetries . . . . . . . . . . 231

Appendix 11.A Hamiltonians by Substitution? 233

Chapter 12 The Harmonic Oscillator and Fields 237

12.1. The Schrödinger Equation for SHM . . . . . . . . . . . . 237

12.2. SHM Details . . . . . . . . . . . . . . . . . . . 239

12.3. Factorisation Method . . . . . . . . . . . . . . 241

12.4. Interpreting the SHM Solutions . . . . . . . . . . . 242 
12.5. Vibrations of Fields and "Particles" . . . . . . . . . . . 244

12.5.1. Phonons and Photons . . . . . . . . . . . . . . 248

12.6. Second Quantisation . . . . . . . . . . . . . 249

Chapter 13 Perturbation Theory and Epicycles 251

13.1. Perturbation Theories in General . . . . . . . . . . . . 251

13.2. Perturbed Schrödinger Equations . . . . . . . . . . 252

13.3. Polarisation of Electron Distribution . . . . . . . . . . 255

13.4. Interpretation of Perturbation Theory . . . . . . . . 256

13.5. Quantum Theory and Epicycles . . . . . . . . . . 258

13.6. Approximations to Non-existent Functions . . . . . . . . . 259

13.7. Summary for Perturbation Theory . . . . . . . . . . . 261

Chapter 14 Formalisms and "Hidden" Variables 263

14.1. The Semi-empirical Method . . . . . . . . . . . . . 263

14.2. The Chemical Bond . . . . . . . . . . . . . . . . . . . 264

14.3. Dirac's Spin "Hamiltonian" . . . . . . . . . . . . . . . 267

14.4. Interpretation of the Spin Hamiltonian . . . . . . . . . . 268

$\begin{array}{lll}\text { Part } 6 & \text { Disputes and Paradoxes } & 271\end{array}$

Chapter 15 Measurement at the

Microscopic Level 273

15.1. Recollection: Concrete and Abstract Objects . . . . . . . . 273

15.2. Statistical Estimates of Probabilities . . . . . . . . . 275

15.2.1. von Neumann's Theory of Measurement . . . . . . 278

15.3. Measurement as "State Preparation" . . . . . . . . . . . . . 281

15.4. Heisenberg's Uncertainty Principle . . . . . . . . . . . . . 284

15.4.1. Measurement and Decoherence . . . . . . . . . . 286

15.5. Measurement Generalities . . . . . . . . . . . . . . 287

Appendix 15.A Standard Deviations of

Conjugate Variables

Chapter 16 Paradoxes 291

16.1. The Classical Limit . . . . . . . . . . . . . . . . . . . . . . . 291

16.1.1. The Ehrenfest Relations . . . . . . . . . . . . . 293

16.2. The Einstein-Podolsky-Rosen (EPR) Paradox . . . . . . . . 294

16.2.1. The EPR Original . . . . . . . . . . . . . 295 
16.2.2. Bohm's Modification . . . . . . . . . . . . 297

16.2.3. Bell's Inequality and Theorem . . . . . . . . . . 298

16.3. Bell's Assumptions . . . . . . . . . . . . . . . . 300

16.3.1. Lessons from EPR . . . . . . . . . . . . . . . . 303

16.3.2. Density of Spin and EPR . . . . . . . . . . . 304

16.4. Zero-Point Energy . . . . . . . . . . . . . . 307

Chapter 17 Beyond Schrödinger's Mechanics? 311

17.1. An Interregnum? . . . . . . . . . . . . . . . . 311

17.2. The Avant-Garde . . . . . . . . . . . . . 313

17.3. The Break with the Past . . . . . . . . . . . . . . . 314

17.4. Classical and Quantum Mechanics . . . . . . . . . . 315

$\begin{array}{ll}\text { Index } & 319\end{array}$ 


\section{Preface}

The presentation and interpretation of (non-relativistic) quantum mechanics is a very well-worked area of study; there have to be very good reasons for adding to the literature on this subject.

My reasons are (obviously) that I am far from satisfied with much of the published work and find difficulties with some points, in particular:

- Any abstract formalism is much less rich than the structure from which it has been abstracted; a fact that even Wittgenstein had to come to terms with in the latter part his adult life.

Language is richer than (cannot be reduced to) a representation of logic, Schrödinger's mechanics is richer than (cannot be reduced to) a representation of Hilbert space. Just as language contains more structures than logic so Schrödinger's mechanics contains more structures than those of Hilbert space.

- The use of probability in quantum theory is arbitrary, eccentric, out of step with modern probability theory and is the source of the majority of "paradoxes" in the interpretation of quantum theory. While these paradoxes are the bread and butter of some of the more popular expositions of quantum theory, I cannot say that I am fond of paradoxes in physical theories.

- Although positivism is discredited as a philosophy of science it has left a huge clutter of verbal and conceptual debris strewn across the field of quantum theory. Positivism in its most aggressive form (instrumentalism) makes the mistake of confusing the meaning of a concept with the way in which numerical values of the variables involved in that concept may be determined. This attitude has, of course, added to the confusion about probabilities; "defining" them in terms of the ways in which they might be measured thus reinforcing the view that probabilities are 
frequency ratios and the everyday opinion that probabilities are applicable to individual events.

- More mundanely, the prescriptions for generating Schrödinger's mechanics from classical mechanics in the vast majority of texts are wrong; they simply do not work. We are saved from total chaos by the fact that the form of the Schrödinger equation is known and used independently of these formal prescriptions by working scientists. I am at a loss to explain why this central point is ignored in text after text, both on the applications of quantum theory and on its interpretation.

Most modern studies of the interpretation of quantum mechanics are "modern" in another sense; that used in literary criticism. They study the theory of quantum mechanics rather than the quantum mechanics of the energetics and distribution of sub-atomic particles. Many of these works concentrate on the alleged consequences of imaginary experiments involving spin angular momentum; uncritically using the colloquial, "everyday" interpretation of probability rather than the modern mathematical theory. Indeed, scarcely any text says what is meant by "probability", leaving the reader to assume that the everyday interpretation is correct. If a Hamiltonian is used at all in these expositions it is an empirical one using "coupling constants" and making no reference to the laws of interaction in physical systems.

In contrast, I wish to present a study of what I have called Schrödinger's mechanics, the richest, most concrete and most thoroughly interpretable of a variety of more-or-less abstract structures falling under the umbrella of "quantum mechanics". Quantum theory is a mathematically articulated theory embedded in a historical stream of scientific thought; it cannot be understood or its interpretation appreciated without addressing these obvious facts. I shall not shrink from joining scientists in cosmology, geology, biology, chemistry, history, archeology, sociology, paleontology, (even physics) in using, where possible, a mechanistic mode of explanation occasionally involving unobserved, even unobservable, objects and processes. What is more, I will occasionally look at actual solutions of the (spatial differential) equations to examine how they are capable of interpretation and how this interpretation bears on the referent of Schrödinger's mechanics.

In a previous work I chose ${ }^{1}$ to express myself using the "ensemble" interpretation of probabilistic statements since this interpretation has been

\footnotetext{
${ }^{1}$ With considerable reservations, as I explained in an Appendix to the work.
} 
one of the major strands in quantum theory (notably by Einstein) and because it is closest to the "abstract object" interpretation which I use in this work. My main aim was twofold: to stay in contact with one of the main streams of thought in quantum theory and, more important, to emphasise the incorrectness of the colloquial interpretation of probability which assumes that probability statements apply to single concrete systems. I now regret this, since even the ensemble interpretation, although vastly superior to the colloquial interpretation, cannot be made precise enough for my purposes here.

Two of the main lessons of the past century in the investigation of the structure of mathematics and, in general, of mathematically articulated theories have been the failures of ordinary logic and intuition for:

- Systems with an infinite number of members or for infinite combinations of statements,

- Constructions which are self-referential like the ones which generate Russell's paradox or for systems whose logic may be "internalised" like Gödel's theorem.

So, generally speaking, I shall not be concerned with "the wave function of the Universe", "the wave function of the measuring apparatus" or even "the wave function of the observer" since the first is both recursively selfreferential and with infinite referent and the others arguably so. I shall, therefore, try to avoid writing down symbols for non-existent mathematical objects; the typographical equivalent of hot air. The science of the very small and very light is quite complex enough for me, I do not have time (literally or figuratively), or indeed the expertise, to express opinions about the bearing of quantum theory on God, Mind or Consciousness.

My aim is entirely bourgeois in the sense used by Wittgenstein and by Marx. I try to solve the problems of Schrödinger's mechanics "from within"; I do not wish to sweep away quantum theory but to bring this most successful physical theory of the twentieth century back into the mainstream of the great tradition of the mechanics of Newton, Lagrange and Hamilton and to base its interpretation on the work of a pillar of twentieth century mathematics: A. N. Kolmogorov.

The degeneration of the interpretation of modern science into silopsism and triviality requires an entirely different kind of investigation for which I have neither the qualifications nor the stomach. 
This page is intentionally left blank 


\section{Organisation}

As this work is presented in a rather unusual way, this short chapter gives both a description of the organisation of material and an attempted justification for this choice.

Analysis and interpretation of Schrödinger's mechanics is a very "mature" subject in the sense that an enormous amount has been written about the matter without any consensus emerging. Or, rather, such consensus as there is about the interpretation of quantum theory is, in my view, based on an erroneous (pre-Kolmogorov) view of probability. So, in what is written here almost every page (indeed, in places, almost every sentence) will contain material that is in dispute. Using Schrödinger's starting point and Kolmogorov's probability theory, I shall say (and I shall prove), among other things, that:

- Probability statements do not refer to individual objects

- Probability distributions do not "collapse"

- The Hamiltonian operator cannot be obtained by an operator substitution in the classical Hamiltonian function

- Dynamical variables may or may not all be represented by linear operators but there is no general way to find the form of these operators

- Conserved quantities are not always represented by operators which mutually commute

- It is nonsensical to give a physical interpretation to the terms in a perturbation expansion

To take the time and space to discuss the opposing views on every such point is tiresome and, more importantly, makes the presentation diffuse and lacking in direction. I shall therefore simply give my presentation 
of probability and Schrödinger's mechanics without detailed references to other points of view or, indeed, in most cases even giving an opposing view a "fair hearing".

This device will enable the presentation of the theory and interpretation of Schrödinger's mechanics "as if" one were developing an interpreted theory $a b$ initio in the cleanest and most logical manner while conveniently ignoring other viewpoints. My defence for this outrageous choice is twofold: defending my position would simply take too much space and the alternative and opposing views are too well-known to need rehearsing here.

Throughout the work I use the term "state function" rather than the familiar "wave function"; this is a deliberate attempt to avoid, even in terminology, the celebrated wave/particle duality. In fact the solutions of the Schrödinger equation are only waves in the extremely atypical case of a particle in the absence of a field of force (a "free" particle). And even these waves are not real physical waves but particular forms of a function which generates a probability distribution. I have also tended (as I say on page 148 of Chapter 8) to work in a system of units - "atomic units" in which Planck's constant has the value unity unless the historical context explicitly demands the appearance of of $h$. 